\begin{tabular}{|c|c|c|c|c|c|}
\hline MUNIBE Antropologia-Arkeologia & $n^{\circ} 71$ & $181-191$ & DONOSTIA & 2020 & ISSN 1132-2217 • elSSN 2172-4555 \\
\hline
\end{tabular}

\title{
La población altomedieval de Sta. María de Tejuela (Bozoó, Burgos). Notas paleodemográficas
}

\author{
The high-medieval population of Sta. María de Tejuela \\ (Bozoó, Burgos). Paleodemographic notes
}

PALABRAS CLAVES: Paleodemografía, Edad Media, Bioarqueología, fertilidad, índice de juventud GAKO-HITZAK: Paleodemografia, Erdi Aroa, Bioarkeologia, ugalkortasuna, gaztetasun-indizea. KEY WORDS: Paleodemography, Middle Age, Bioarcheology, fertility, juvenile index.

\section{Sylvia A. JIMÉNEZ-BROBEIL*, Rosa M. MAROTO BENAVIDES(1)} María G. ROCA ${ }^{(1)}$, José F. MARTíN-ALONSO(1)

\section{RESUMEN}

Se analizan los datos que proporciona el estudio antropológico y arqueológico del cementerio altomedieval de Santa María de Tejuela (Bozoó, Burgos) que pueden aportar alguna información sobre aspectos paleodemográficos. El número de individuos exhumados asciende a 182, aunque solo se ha podido asignar edad y sexo a 125 de ellos. El número de individuos infantiles y juveniles es reducido, lo que proporciona un índice de juventud muy bajo. Entre los adultos los resultados sobre la mortalidad son los propios de una población rural medieval que vivió bajo duras condiciones de vida, sobre todo las mujeres. La mayoría de enterramientos de adultos sugiere una población con una aparente baja fertilidad.

\section{LABURPENA}

Santa María de Tejuelan (Bozóo, Burgos) kokatutako Goi Erdi Aroko hilerrian egindako ikerketa antropologiko eta arkeologikoak ematen dituen datuak aztertu dira alderdi paleodemografikoen inguruan zer informazio eman dezaketen jakiteko. Orain arte osotara 182 pertsona deshobiratu dituzte bertan, baina horien artean soilik 125 pertsonaren adina eta sexua identifikatu ahal izan dira. Haur eta gazte gutxi aurkitu direnez, gaztetasun-indizea oso baxua da. Gazteen artean, hilkortasunaren inguruan lortu diren emaitzak bizi-baldintza gogorretan (batez ere emakumeak) bizi izan zen Erdi Aroko landa-eremuko biztanlerien parekoak dira. Hobiratze gehienak helduenak izateak bertako biztanleriak ugalkortasun baxua zuela iradokitzen du.

\section{ABSTRACT}

This paper analyzes the data provided by the anthropological and archaeological study of the Santa María de Tejuela cemetery (Bozoó, Burgos), which may provide some information on paleodemographic aspects, bearing in mind their difficult and sometimes contradictory interpretation. The number of individuals determined from the skeletal remains amounts to 182. However, the number of skeletons with age and sex assigned is 125. This assignment has been carried out by a team of three researchers to minimize inter-observer bias in the application of the different methods utilized. Regarding the distribution by sex, including the three juvenile individuals, males account for the majority of individuals (56.7\%) compared to females (43.3\%). Among the latter, the majority died before reaching the age of 41 and no woman lived beyond 61 years. The number of children and youth burials is low in relation to that of adults, and the estimated youth index is very low $(0.10)$ and significantly lower than that of other contemporary populations in the region. Non-adults show a very high mortality in the first year of life, somewhat lower in the following two years and with a significant drop from the third year on. The mortality results of adults are consistent with those of a medieval rural population, with low average life expectancy, especially among women. The paucity of infant and youth burials, which provides a mortality profile that does not follow the expected U-shape of pre-industrial populations, cannot be attributed exclusively to taphonomic or methodological factors. Everything points to a population with low fertility, in which there could have been emigrants who did not form families in the place, which could fit with the instability of life in a border area.

\footnotetext{
(*) Departamento de Medicina Legal, Toxicología y Antropología Física. Facultad de Medicina. Avda de la llustración 9, torre A, planta 4. 18016 GRANADA (España). E-mail: jbrobeil@ugr.es.

(1) Área de Antropología Física. Universidad de Granada
} 


\section{INTRODUCCIÓN}

Un cementerio altomedieval que ha vuelto a ponerse de actualidad gracias a una nueva excavación y a una serie de trabajos científicos, algunos todavía en curso, es el de Santa María de Tejuela (Bozoó, Burgos), conocido en la historiografía con el nombre de Villanueva de Soportilla (Aratikos Arqueólogos, 2010; Martín Alonso et al., en prensa; Palomino y Negredo, 2020). Las primeras campañas de excavaciones fueron dirigidas por el profesor Alberto del Castillo (1972), uno de los fundadores de la Arqueología medieval española, quien siguió las teorías del historiador Claudio Sánchez-Albornoz (1966). En este sentido, del Castillo vinculó la fundación del poblado asociado a este cementerio con el movimiento de repoblación ultramontana del reino astur-leonés durante el reinado de Alfonso III de Castilla (866-904 A.D). También definió este tipo de cementerios caracterizado por una mayoría de tumbas excavadas en roca. Posteriormente, se han planteado otras teorías sobre estos cementerios desde diferentes perspectivas como considerarlos centros de poder o delimitación de territorios (López et al., 2016; Martín Viso 2012; Padilla y Álvaro 2010 y 2013; Quirós 2006; Tente 2015).

El cementerio Sta. María de Tejuela (Tejuela, en adelante) pertenece a lo que Padilla y Álvaro (2010) denominan como "grandes necrópolis", es decir, conjuntos de enterramiento de comunidades aldeanas estables con más de cien tumbas situadas en torno a un centro de culto. Se ha identificado el área de habitación que permanece sin excavar y la totalidad del espacio cemente- rial donde se conserva la planta de la iglesia y un edículo que debió formar parte de un importante edificio del que no se ha podido determinar si tendría carácter civil o religioso (Palomino y Negredo, 2020). La mayoría de las tumbas están excavadas en la roca, con predominio de las antropomorfas y de las de forma de bañera, si bien figuran muchas construidas con lajas de piedra. En este sentido el conjunto es muy similar a los cementerios burgaleses de Cuyacabras, Revenga o Regumiel y al soriano de Duruelo (Álvaro et al., 2018; Padilla y Álvaro, 2010 y 2013; López et al., 2016). De momento, se dispone de 11 dataciones absolutas obtenidas a partir de restos esqueléticos cuyos extremos marcan un rango que va desde finales del siglo VIII a comienzos del XI, pero con un periodo central de utilización de mediados del IX al final del X (Martín Alonso et al., en prensa). Tejuela resulta un caso especial porque es uno de los pocos cementerios altomedievales del Norte peninsular que ha proporcionado numerosos restos humanos como pueden ser los casos de Dulantzi, Treviño o Marialba de Ribera entre otros (Candelas González et al., 2016; Fernández Crespo, 2011; Quirós, 2011).

Todos estos cementerios se han analizado desde la perspectiva holística de la Bioarqueología, entre cuyos objetivos figura el estudio de esqueletos humanos para obtener información sobre los aspectos demográficos del grupo que habitó un territorio. Dichos aspectos se relacionan con factores biológicos, sistemas socioeconómicos, características medioambientales, etc. (Chamberlain, 2006). Sin embargo, los estudios paleodemográficos son muy complicados de realizar porque, a diferencia de la demografía histórica, que se

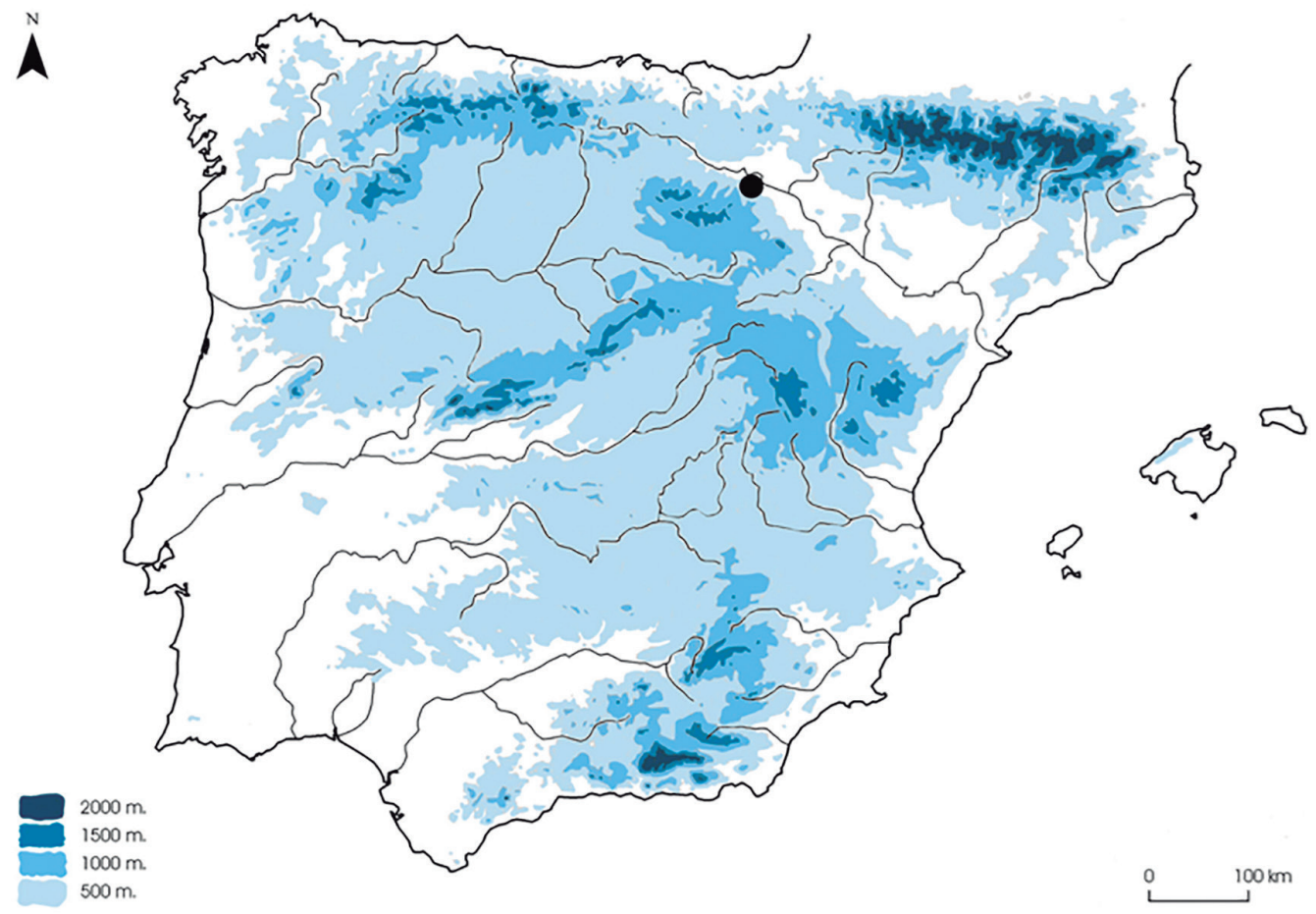

Fig.1. Mapa de la Península lbérica con la localización del yacimiento de Sta. María de Tejuela. / Map of the Iberian Peninsula with the location of the site of Sta. María de Tejuela. 
basa en fuentes documentales como registros civiles, libros parroquiales o censos, solo se cuenta con los esqueletos hallados en las excavaciones arqueológicas. Independientemente de los problemas intrínsecos a ellos como la conservación o la fiabilidad de la estimación del sexo y edad, de los que se habla más abajo, el primer muro con el que choca la Paleodemografía es que estudia muestras de individuos en el momento de su fallecimiento, que no reflejan a una población viva. El segundo escollo viene dado por las asunciones de los diferentes métodos usados. Tanto la elaboración de las clásicas tablas de vida (Acsádi y Nemeskéri, 1970), como la aplicación de tablas modelo basadas en poblaciones preindustriales (Séguy et al., 2006) parten de la premisa de que la población en estudio se mantuvo estacionaria, sin crecimiento, ni crisis de mortalidad ni movimientos migratorios. Si se acepta la hipótesis de que las poblaciones antiguas y medievales fueron similares a las preindustriales (Séguy et al., 2006), se caracterizarían por una alta fertilidad, baja esperanza media de vida al nacer, elevada mortalidad infantil y un crecimiento prácticamente igual a cero. Sin embargo, las poblaciones preindustriales estuvieron sometidas a crisis de mortalidad que conllevaban una muy importante pérdida de vidas, seguida de un incremento notable de la fecundidad que permitía el restablecimiento en un tiempo relativamente rápido del tamaño efectivo de la población (Livi-Bacci, 1999). Por otra parte, un modelo de población estacionaria no tendría en cuenta las migraciones estacionales, que podrían ser muy frecuentes en áreas rurales, ni la urbanización y construcción de nuevos asentamientos (Barbiera y DaIla-Zuanna, 2009). Como se puede ver, cualquier es- tudio paleodemográfico es meramente aproximativo y solo permite obtener datos muy sesgados y ello teniendo muy en cuenta los procesos sociales, económicos e históricos que dieron lugar a una muestra esquelética (Milner et al., 2008).

Dicho esto, el objetivo de este trabajo es analizar los datos que proporciona el estudio antropológico y arqueológico del cementerio de Tejuela que puedan aportar alguna información sobre aspectos demográficos de la población que se enterró en él teniendo muy en cuenta la difícil y a veces contradictoria interpretación de los mismos (Séguy et al., 2008).

\section{MATERIAL Y MÉTODOS}

Según el inventario efectuado por Aratikos Arqueólogos (2010) en el cementerio de Tejuela figuran 340 tumbas documentadas (figura 2), pero no suponen la totalidad del cementerio puesto que en el cuadrante $\mathrm{NO}$ del mismo se ha reservado una zona de unos 200 $\mathrm{m}^{2}$ sin excavar. Tanto en esta excavación como en la de Alberto del Castillo (1972) muchas tumbas se encontraron vacías. Otras conservaban un esqueleto en posición, con mayor o menor grado de preservación. Tumbas alteradas conservaban esqueletos parciales y en algunas se encontraron huesos sueltos muy posiblemente procedentes del expolio de tumbas cercanas (Aratikos Arqueólogos, 2010). Entre las tumbas documentadas se han dejado 71 sin excavar como reserva. Tanto por el informe de los arqueólogos (Palomino y Negredo, 2020) como por los análisis antropológicos (Maroto, 2004; Martín Alonso, 2018; Souich et al., 1991)

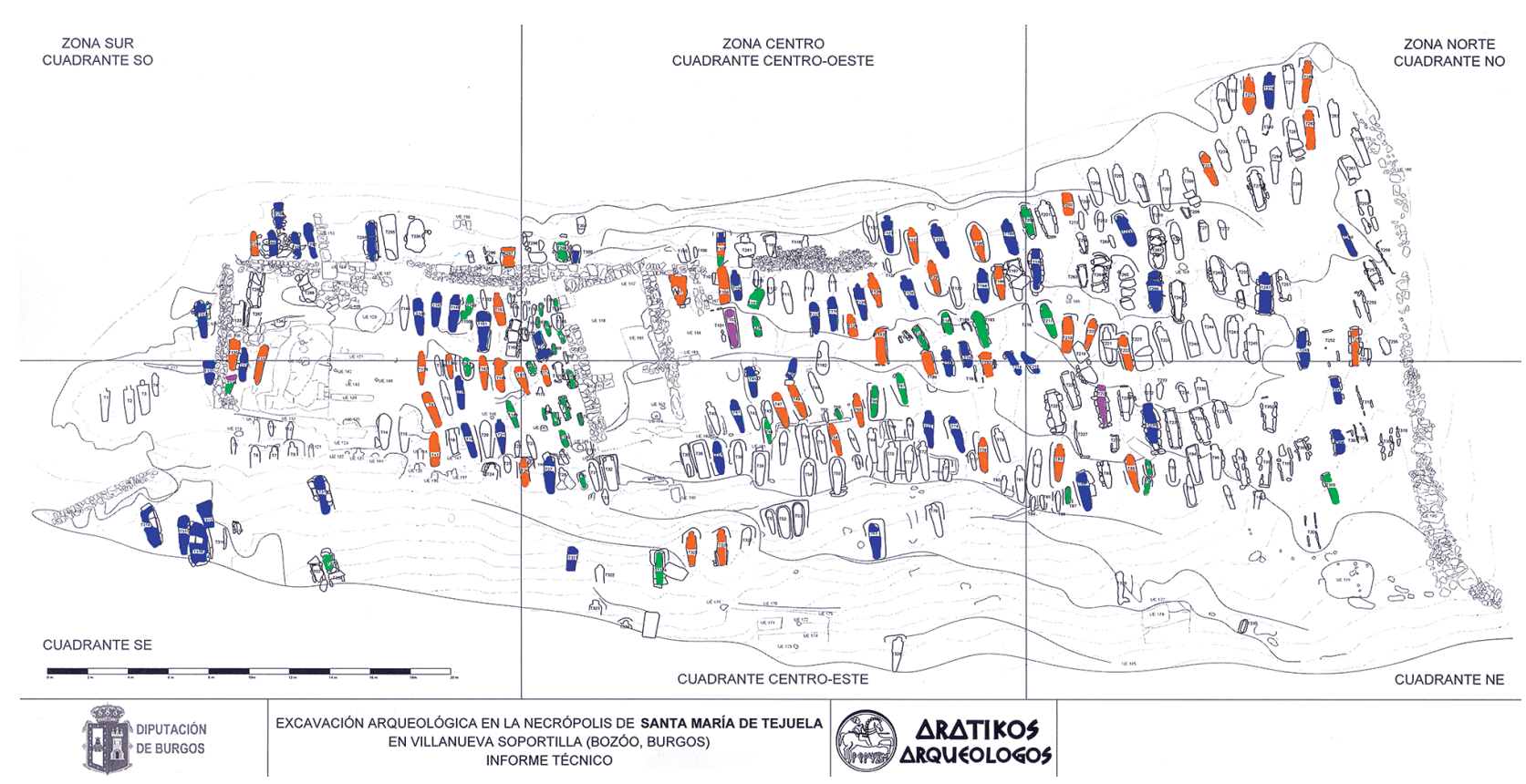

Fig.2. Plano del yacimiento. En azul: tumbas con varones; en naranja: tumbas con mujeres; en violeta: tumbas con adultos indeterminados; en verde: tumbas con subadultos. / Map of the site. In blue: male burials; in orange: female burials; in violet: indeterminate adult burials; in green: non-adult burials. 
hay un acuerdo de que no se produjeron reutilizaciones de tumbas. En los ocasionales casos en los que se han documentado restos de dos individuos en una tumba no se trata de reacomodos sino de algún hueso suelto situado desordenadamente sobre un esqueleto yacente en una tumba sin cubierta y próxima a otras expoliadas. Así pues, en principio cada tumba indicaría un solo individuo.

En los estudios antropológicos arriba mencionados el número mínimo de individuos determinado a partir del estudio de los restos óseos asciende a 182, es decir, equivaldría al 53.5\% de la muestra si admitimos un total de 340 individuos/tumbas. Sin embargo, el número de esqueletos a los que ha podido asignarse la edad y el sexo (en este caso solo para los mayores de 13 años) es de 125, lo que supondría que solo se contaría con un $36.7 \%$ del hipotético total de 340 individuos/tumbas. Con ello se tropieza con uno de los primeros problemas en estudios paleodemográficos: si la muestra es representativa de la población (Jackes, 2011; Wright y Yoder, 2003). De hecho, estos esqueletos ni siquiera son una muestra fiable, dado que no son una selección al azar de aquellos que una vez estuvieron vivos (Waldron, 2007). Además, por muy bien excavado que esté un cementerio, no es lo mismo que una comunidad viva y las conexiones entre una población pasada y una colección esquelética son complejas y tortuosas (Milner et al., 2008). Un cementerio muestra la mortalidad (Jackes, 2011) y los esqueletos representan las malas condiciones de salud de las personas en el momento de su fallecimiento y además están sometidos a la paradoja osteológica (Saunders y Hoppa, 1993; Waldron, 1994; Wright y Yoder, 2003).

Aunque estaba estimada la edad y sexo de estos 126 individuos, se ha vuelto a revisar por un equipo de tres investigadores para minimizar la parcialidad inter observadores en la aplicación de los diferentes métodos usados. Para la estimación de la edad de los individuos inmaduros se han tenido en cuenta la erupción dental, la longitud de las diáfisis y la soldadura de las epífisis (Buikstra y Ubelaker, 1994; Scheuer y Black, 2000). Para los adultos se han empleado la sinostosis de las suturas craneales y los cambios en la sínfisis del pubis, faceta auricular del ilion y extremidad esternal de la tercera costilla (Buikstra y Ubelaker, 1994; Byers, 2005); asimismo se ha tenido en cuenta la presencia de lesiones vinculadas con patología degenerativa. La aplicación de estos métodos ha vuelto a resaltar otros problemas de los estudios paleodemográficos, como que dichos métodos no son coincidentes en sus resultados y están elaborados a partir de series esqueléticas que no tienen nada que ver con las poblaciones medievales del norte de la Península Ibérica (Bocquet-Appel y Bacro, 2008; Milner y Boldsen, 2012; Usher, 2002; Wittwer-Backofen et al., 2008). En segundo lugar, los métodos muestran rangos de edad más cortos para los individuos más jóvenes, pero impiden estimar la edad con precisión de los individuos de edades más avan- zadas que, además, tienden a subestimarse (Hoppa y Vaupel, 2002; Jackes, 2000, 2011; Milner y Boldsen, 2012; Müller et al., 2002; Wittwer-Backofen et al., 2008). Tampoco puede precisarse quien fue la persona que vivió más años y qué edad alcanzó por lo que generalmente en las tablas de vida se ponen de forma arbitraria los topes de los intervalos de edad (Milner y Boldsen, 2012). Otro problema vinculado a la determinación de la edad es el grado de conservación de los esqueletos que impide que los métodos seleccionados se puedan aplicar a todos los individuos. El grado de conservación es además una de las principales causas de sesgo por edad en los estudios paleodemográficos (Bello et al., 2006; Wittwer-Backofen et al., 2008).

Con todas estas premisas se han distribuido los esqueletos en las siguientes categorías de edad: menores de 1 año, de 2 a 6 años, de 7 a 12 años, de 13 a 20 años, de 21 a 40 años, de 41 a 60 años y mayores de 61 años. Los no adultos se han subdividido a su vez según su edad precisa entre los grupos siguientes: menores de un año, de 1 a 3 , de 4 a 6 , de 7 a 9 , de 10 a 12 y de 13 a 15 .

En cuanto a la determinación del sexo se ha utilizado la morfología de pelvis y cráneo (Buikstra y Ubelaker, 1994). En este sentido el grado de conservación también es determinante porque ha obligado a descartar del estudio a los esqueletos que no conservaban buena parte de estas regiones anatómicas.

Dadas las características de las tumbas de este cementerio, en su mayoría excavadas en la roca, se han tenido en cuenta las dimensiones de las mismas para tener una idea aproximada sobre el ritual funerario seguido con los individuos no adultos y su distribución por el cementerio. En un trabajo anterior (Martín Alonso et al., en prensa) se analizaron las relaciones existentes entre la edad y las longitudes de las tumbas. En el caso concreto de los esqueletos infantiles se comprobó que todos los fallecidos antes de cumplir un año se situaban en tumbas con longitudes inferiores a $80 \mathrm{~cm}$. En tumbas con longitudes entre 100 y $130 \mathrm{~cm}$ aparecían individuos fallecidos antes de cumplir 6 años, pero también alguno con 7-8 años e incluso uno juvenil de talla muy baja. En esta ocasión y asumiendo todos los riesgos del procedimiento, se han tenido en cuenta las tumbas menores de $80 \mathrm{~cm}$, las comprendidas entre 81 y 110 $\mathrm{cm}$ y las que miden entre 111 y $150 \mathrm{~cm}$, presumiblemente atribuibles a individuos no adultos de más de 7 años. Del total de 340 tumbas solo 236 proporcionan la longitud completa con exactitud (Aratikos Arqueólogos, 2010) y son las que se han utilizado para este análisis.

Se ha calculado el denominado índice de juventud $(\mathrm{IJ})\left({ }_{15} \mathrm{P}_{5}\right)$ o ratio de individuos fallecidos entre 5 y 15 años en relación a los fallecidos con más de 5 (Bocquet-Appel, 2002; Bocquet-Appel y Naji, 2006) en base a la fórmula $\mathrm{d}=\mathrm{D}_{5-15} / \mathrm{D}_{5+}$. Esta ratio es muy usada en estudios paleodemográficos porque minimiza la falta de niños pequeños y la dificultad de estimar con precisión 
la edad de los adultos (Barbiera y Dalla-Zuanna, 2009). También se ha elaborado una tabla de vida (Acsádi y Nemeskéri, 1970) para los individuos adultos según sexo y clase de edad para poder realizar comparaciones con otras poblaciones analizadas con los mismos métodos.

\section{RESULTADOS}

La distribución según categoría de edad y sexo de los 125 individuos incluidos en este estudio se expone en la tabla I. Los individuos menores de 7 años suponen el $18.4 \%$ de la muestra, cifra muy baja que impide el cálculo, en cualquier caso siempre aproximativo, de la esperanza media de vida al nacer.

\begin{tabular}{|c|c|c|c|c|c|}
\hline Edad & $\mathbf{N}$ & $\%$ & \multicolumn{3}{|c|}{ Sexo } \\
\hline & & & Indeterminado & Masculino & Femenino \\
\hline$<1$ año & 11 & 8.8 & 11 & - & - \\
\hline 1-6 años & 12 & 9.6 & 12 & - & - \\
\hline 7-12 años & 5 & 4.0 & 5 & - & - \\
\hline 13-20 años & 3 & 2.4 & - & 2 & 1 \\
\hline 21-40 años & 56 & 44.8 & - & 29 & 27 \\
\hline 41-60 años & 35 & 28.0 & - & 21 & 14 \\
\hline$>61$ años & 3 & 2.4 & - & 3 & 0 \\
\hline Total & 125 & & & 55 & 42 \\
\hline
\end{tabular}

Tabla 1: Distribución según categoría de edad y sexo de los individuos seleccionados para este estudio. / Distribution according to age category and sex of the individuals selected for this study.

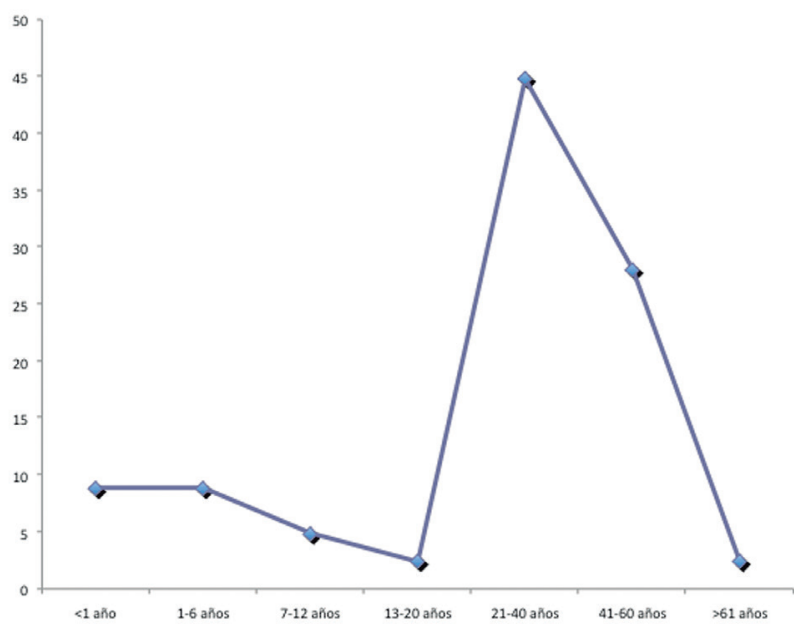

Fig.3. Distribución de los esqueletos analizados según clases de edad. / Distribution of the skeletons analyzed according to age classes.

La falta de individuos fallecidos en la primera infancia en relación a los fallecidos en la edad adulta se refleja en el perfil de la figura 3 donde se hubiera esperado una curva en forma de $U$, propia de la mortalidad de poblaciones del antiguo régimen demográfico. El arranque de este perfil esperado, sin embargo, sí se aprecia en la curva de mortalidad de los individuos inmaduros (figura 4) donde destacan los porcentajes mucho más elevados de los fallecidos antes de cumplir un año y de los fallecidos entre 1 y 3 .

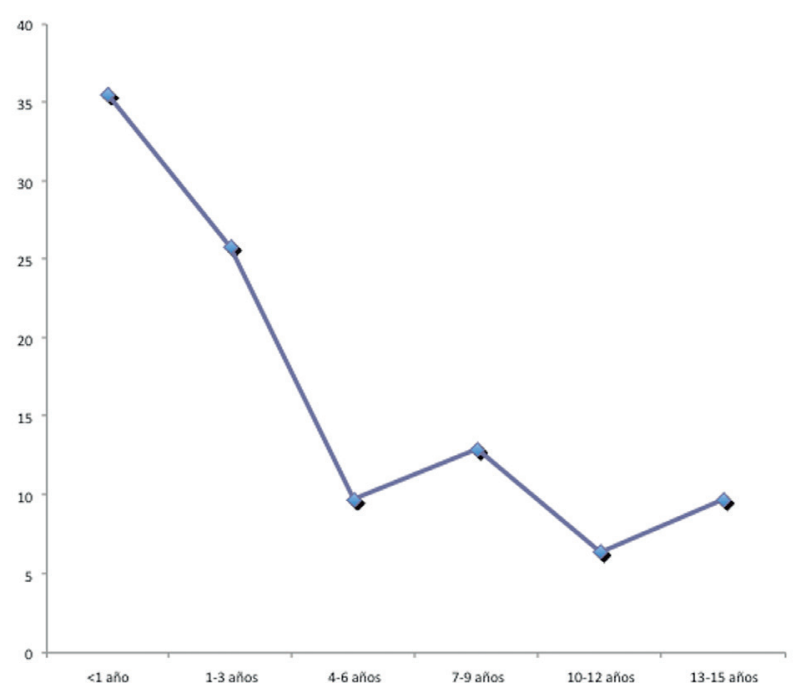

Fig.4. Distribución porcentual de los esqueletos de individuos no adultos. Percentage distribution of the skeletons of non-adult individuals.

La distribución de las longitudes de las tumbas y su equivalente con la edad estimada en los restos óseos se expresa en la tabla II.

\begin{tabular}{|c|c|rr|rr|rr|}
\hline & & \multicolumn{2}{|c|}{ Esqueletos } & \multicolumn{2}{|c|}{ Tumbas } & & \\
\hline $\begin{array}{c}\text { Clase } \\
\text { edad }\end{array}$ & $\begin{array}{c}\text { Longitud } \\
\text { tumbas }\end{array}$ & $\mathrm{N}$ & $\%$ & $\mathrm{~N}$ & $\%$ & $\chi^{2}$ & $\mathrm{p}$ \\
\hline$<1$ año & $<80 \mathrm{~cm}$ & 11 & 8.8 & 28 & 11.8 & 0.51 & 0.47 \\
\hline $1-6$ años & $80-110 \mathrm{~cm}$ & 12 & 9.6 & 32 & 13.5 & 0.85 & 0.35 \\
\hline $7-15$ años & $110-150 \mathrm{~cm}$ & 8 & 6.4 & 21 & 8.9 & 0.39 & 0.53 \\
\hline$>20$ años & $>150 \mathrm{~cm}$ & 94 & 75.2 & 155 & 65.7 & 3.03 & 0.08 \\
\hline
\end{tabular}

Tabla 2: Frecuencias de distribución de esqueletos por clase de edad y longitud de tumbas equivalentes y comparación estadística. / Skeletal distribution frequencies by age class and length of equivalent graves and statistical comparison.

El conjunto de tumbas con longitudes atribuibles a menores de 7 años equivale al 25.4\%., valor también inferior al esperado para el número de fallecidos en este segmento de edad en una población del régimen demográfico antiguo. Sin embargo, ambas distribuciones (figura 5) son bastante similares (no se alcanza la significación estadística en ninguna de las clases de edad/categoría de longitud) y la curva de mortalidad realizada según las longitudes de las tumbas tampoco sigue un modelo en $U$.

El parámetro del IJ $\left({ }_{15} \mathrm{P}_{5}\right)$ calculado es de 0.10. Si se aplica la misma fórmula al segmento de longitudes de tumba entre 110 y $150 \mathrm{~cm}$ con relación a las comprendidas entre $110 \mathrm{~cm}$ en adelante, el valor obtenido es 0.12 , muy similar al que proporcionan los restos esqueléticos. 


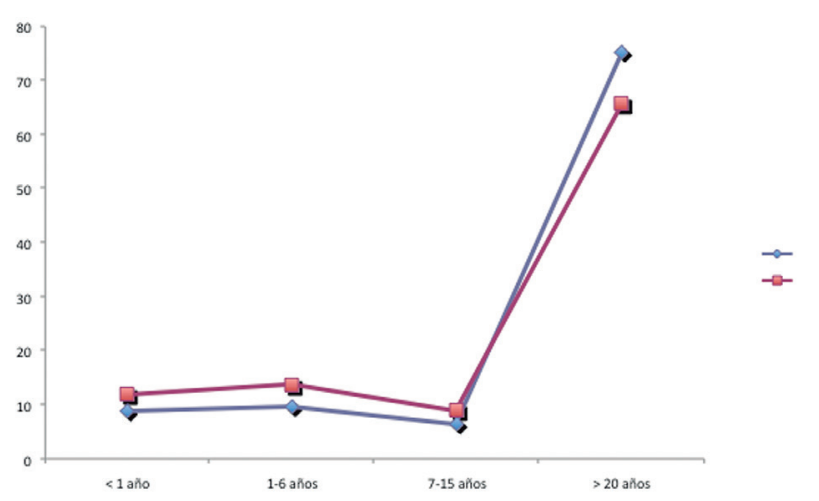

Fig.5. Comparación de la distribución de los esqueletos por clases de edad (línea azul) y de las tumbas cuyas longitudes son equivalentes a dichas clases (línea roja). / Comparison of the distribution of skeletons by age classes (blue line) and of graves whose lengths are equivalent to those classes (red line).

En cuanto a la mortalidad de los adultos la gráfica (figura 6) muestra una mayor incidencia en el segmento comprendido entre los 21 y 40 años, puesto que estos suponen el $44.8 \%$ del total de los fallecidos y el $59.6 \%$ del total de los mayores de 21 años. Los fallecidos entre los 41 y 60 años equivalen, respectivamente al $28.0 \%$ y al $37.2 \%$. Los individuos seniles, fallecidos con más de 61 años, tan solo suponen el 2.4\% y $3.2 \%$. Esta distribución puede apreciarse de forma gráfica en el tercer bloque de columnas de la figura 6. Las diferencias porcentuales entre las dos primeras categorías alcanzan la significación estadística $\left(\chi^{2}=8.79\right.$; $\mathrm{p}<0.001)$, al igual que entre la segunda y la tercera $\left(\chi^{2}=24.8 ; p<0.001\right)$.

En cuanto a la distribución por sexos, incluyendo los tres individuos juveniles, los varones son mayoría $(56.7 \%)$ frente a las mujeres (43.3\%), lo que supone un índice de masculinidad de 1.31, aunque no se alcanza la significación estadística $\left(\chi^{2}=2.97 ; p=0.08\right)$. Entre los adultos menores de 40 años este índice es de 1.07 y se eleva entre los mayores de 41 años a 1.71. Con relación al sexo y edad, entre los varones son mayoría los fallecidos antes de cumplir los 41 años, pero su porcentaje (ver tabla III) no difiere en exceso de los fallecidos entre 41 y 60 años. Sin embargo, los tres varones que alcanzaron la categoría de seniles, sí suponen un porcentaje muy pequeño respecto a la categoría anterior. Esta distribución puede observarse de forma gráfica en las primeras columnas de la figura 6. En la tabla III se expone la esperanza media de vida calculada para cada una de las categorías de edad. Como el intervalo de edad fijado para la última es de 20 años, su valor $e^{\circ} x$ es de 10. En cuanto a las mujeres, la mayoría de ellas falleció antes de cumplir 41 años y este porcentaje (ver tabla III) es significativamente superior al de mujeres que murieron entre los 41 y los 60 años $\left(\chi^{2}=7.02 ; p<0.001\right)$. Con los métodos aplicados para la estimación de la edad ninguna mujer vivió más de 61 años.

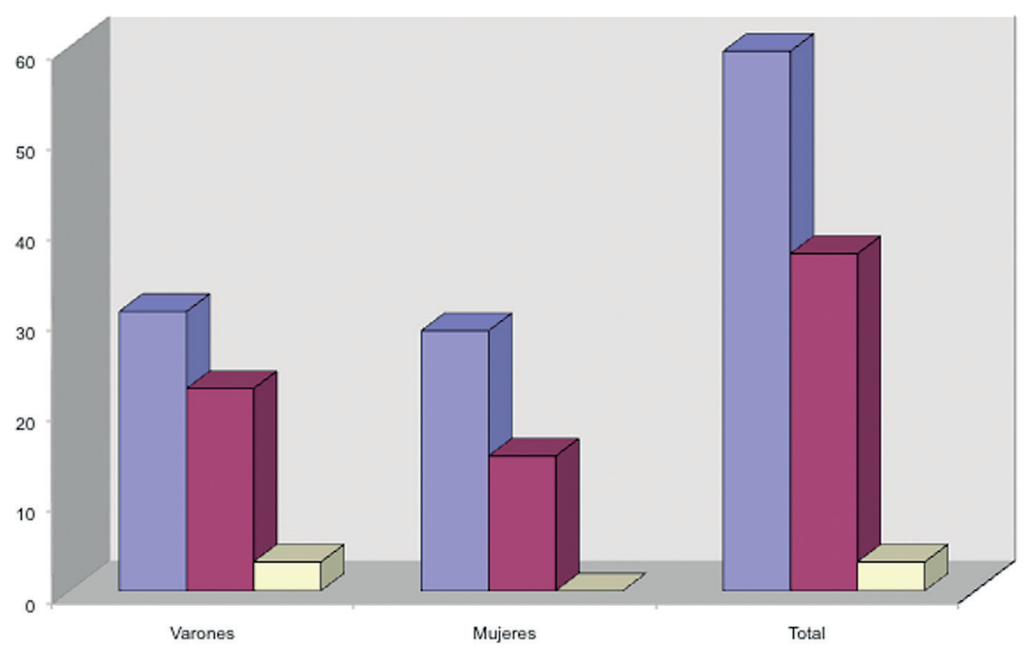

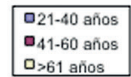

Fig.6. Distribución de los individuos adultos de Tejuela en base a sexo y edad. / Distribution of adult individuals in Tejuela based on sex and age.

\begin{tabular}{|c|c|c|c|c|c|c|c|c|c|c|c|c|c|c|}
\hline Edad & \multicolumn{2}{|c|}{$\mathbf{D}(\mathbf{x})$} & \multicolumn{2}{|c|}{$\mathbf{d}(\mathbf{x})$} & \multicolumn{2}{|c|}{$\mathbf{l}(\mathbf{x})$} & \multicolumn{2}{c|}{$\mathbf{q}(\mathbf{x})$} & \multicolumn{2}{c|}{$\mathbf{L}(\mathbf{X})$} & \multicolumn{2}{|c|}{$\mathbf{T}(\mathbf{X})$} & \multicolumn{2}{|c|}{$\mathbf{E}^{\circ}(\mathbf{X})$} \\
\hline & $\mathrm{V}$ & $\mathrm{M}$ & $\mathrm{V}$ & $\mathrm{M}$ & $\mathrm{V}$ & $\mathrm{M}$ & $\mathrm{V}$ & $\mathrm{M}$ & $\mathrm{V}$ & $\mathrm{M}$ & $\mathrm{V}$ & $\mathrm{M}$ & $\mathrm{V}$ & $\mathrm{M}$ \\
\hline $21-40$ & 29 & 27 & 54.72 & 65.85 & 100.0 & 100.0 & 0.55 & 0.66 & 1452.8 & 1341.5 & 2018.8 & 1683.0 & 20.19 & 16.83 \\
\hline $41-60$ & 21 & 14 & 39.62 & 34.15 & 45.28 & 34.15 & 0.87 & 1.00 & 509.4 & 341.5 & 566.0 & 341.5 & 12.50 & 10.0 \\
\hline$>61$ & 3 & 0 & 5.66 & 0.00 & 5.66 & 0.0 & 1.00 & - & 56.6 & 0.0 & 56.6 & 0.0 & 10.0 & 0.0 \\
\hline & 53 & 41 & & & & & & & & & & & & \\
\hline
\end{tabular}

Tabla 3: Tabla de mortalidad, según sexo y clase de edad de los fallecidos con más de 21 años. $D(x)$ : número de fallecidos por intervalo de edad; d(x): porcentaje de fallecidos; $I(x)$ : porcentaje de supervivientes; $q(x)$ : probabilidad de muerte; $L(x)$ : total de años vividos; $T(x)$ : total de años por vivir; $e^{\circ}(x)$ : esperanza media de vida. / Mortality table, according to sex and age class of the deceased over 21 years old. $D(x)$ : number of deaths by age range; $d(x)$ : percentage of deaths; I $(x)$ : percentage of survivors; $q(x)$ : probability of death; $L(x)$ : total years lived; $T(x)$ : total years to live; $e^{\circ}(x)$ : average life expectancy. 
En conjunto, los varones presentan una mayor esperanza media de vida que las mujeres, tanto por tener representantes que fallecieron en la última categoría de edad como por un porcentaje menor de difuntos entre los 21 y 40 años.

\section{DISCUSIÓN}

El porcentaje de restos esqueléticos de niños menores de 7 años está muy por debajo del 30\% mínimo que se debería esperar en una población arqueológica anterior a la época de difusión de grandes epidemias que afectan especialmente a la infancia como, por ejemplo, la viruela (Lewis, 2007) y al 40\% que estima Buchet (1983) para poblaciones arqueológicas preindustriales. Los fallecidos menores de 8 años (26 casos) suponen el $20.8 \%$, cifra muy inferior al $50 \%$ que estima Livi-Bacci (1999) como la habitual en poblaciones europeas anteriores a la Revolución Industrial con una elevada tasa de mortalidad infantil. Aunque en Tejuela se conservan esqueletos de niños fallecidos antes de cumplir un año - de hecho, figuran incluso dos recién nacidos pretérmino- se repite el fenómeno muy común de la falta de niños pequeños (Chamberlain 2006; Jackes, 2011; Saunders y Hoppa, 1993). La cifra de 25.4\%, calculada a partir las dimensiones de las tumbas, es algo mayor a la que proporcionan directamente los restos esqueléticos lo que demuestra una pérdida de estos por problemas de conservación o excavación deficiente. Sin embargo, ambos métodos de conteo coinciden en que hay pocos individuos infantiles y que el perfil de la mortalidad de la población no sigue el modelo esperado en forma de $U$ que es típico de las sociedades de régimen demográfico antiguo (Jackes, 2011). Los no-adultos, en conjunto, sí siguen este planteamiento en el sentido de que la mortalidad es muy alta en el primer año de vida, baja algo en los dos años siguientes y cae de forma importante a partir del tercer año, tal como era de esperar en un modelo de mortalidad atricional en una población antigua (Chamberlain, 2006; Waldron, 2007).

En conjunto se puede afirmar que, en este cementerio, donde se enterraron incluso niños perinatales, la mortalidad de los individuos inmaduros sigue el modelo esperado de una población de régimen antiguo, pero si se relacionan estos con los adultos, es obvio que en este cementerio faltan enterramientos de niños. En este sentido y dado que se cuenta con la excavación de casi todo el cementerio, surgen dos posibles explicaciones, una de carácter ritual y otra de carácter demográfico. La primera está en relación con el hecho, propio de las comunidades cristianas, de que en el territorio sagrado de un cementerio no pueden enterrarse quienes no sean considerados miembros de la Iglesia. En el caso de los niños muy pequeños surge la pregunta de a qué edad recibían el bautismo y por ende el derecho a ser enterrados en el espacio cementerial comunal. El bautismo de niños pequeños está documentado desde Agustín de Hipona (De peccatorum meritis et remissio- ne et de baptismo parvulorum, I, 26, 39), y en un Sínodo de obispos al que asistió Cipriano de Cartago (1998) se decretó que se podían bautizar los niños a partir del segundo o tercer día del nacimiento. Con esta tradición es normal que figuren dentro del cementerio de Tejuela varios niños fallecidos antes de cumplir un año e incluso perinatales. En muchos cementerios los niños tenían un espacio funerario reservado a ellos (Gélis, 2006) y si este no entró en el perímetro de la zona objeto de la excavación arqueológica, es normal que no aparezcan. En Tejuela el lugar reservado a estos niños, aunque aparecen algunos casos junto a enterramientos de adultos, fue el costado Sur de la iglesia. En otros cementerios medievales del Norte peninsular, como en los de Veranes, Marialba de Ribera, Cuyacabras o Revenga se han delimitado también estos espacios (Candelas González et al., 2016; Padilla y Álvaro, 2010, 2013). Si en Tejuela se enterraron niños de muy corta edad, se han tenido en cuenta todas las tumbas de pequeño tamaño y no hay ningún caso que sugiera que pudieran enterrarse con adultos, la hipótesis del ritual funerario diferencial no explica la escasez de niños muy pequeños.

La explicación de carácter demográfico a la escasez de enterramientos de niños en Tejuela viene dada por una posible menor mortalidad o por una baja fertilidad. Un alto cuidado parental durante el primer periodo de la infancia podría justificar una menor mortalidad que podría llevar aparejada una menor fertilidad (Pfeiffer et al., 2014; Suby et al., 2017). Este fenómeno está atestiguado en series históricas con datos documentales y se ha atribuido a un destete tardío y progresivo (Minvielle, 2007). De cara a evaluar una posible baja fertilidad, imposible de estimar con precisión en una serie arqueológica pequeña (Paine y Harpending, 1996), cobra especial valor el índice de juventud (IJ). Este no tiene en cuenta a los niños muy pequeños sino a los que fallecieron entre los 5 y los 15 años. Tanto el valor obtenido a partir de los restos esqueléticos (0.10) como el calculado a partir de las longitudes de las tumbas (0.12) son bajos y propios de una población en declive. A partir de valores mayores de 0.17 se considera que una población se encuentra en crecimiento (Bocquet-Appel y Naji, 2006). Este crecimiento puede obedecer a un incremento real de la población como el que se produce en países actuales en vías de desarrollo o al proceso de recuperación de efectivos tras una crisis de mortalidad. En cualquier caso, los valores obtenidos son muy pequeños en relación a los de otras poblaciones medievales como la de Marialba de Rivera con un IJ de 0,34 (Candelas González et al., 2016) o la de Veranes con un 0,48 (González Martín et al., 2016). La aparente baja fertilidad señalada en Tejuela puede ser consecuencia de los cuidados parentales y una escasez de brotes epidémicos que causen el fallecimiento de muchos niños y por ello no sea necesaria su rápida sustitución. También puede obedecer a una natalidad controlada que responda a las circunstancias medioambientales y socioeconómicas del entorno y en 
este sentido hay que tener en cuenta que las sociedades agrícolas tendieron a mantener su crecimiento sometido a la limitación de recursos (Flinn, 1989).

Por otra parte, hay que tener presentes las propias características de la población de Tejuela en un entorno de fundación de nuevos asentamientos y movimientos poblacionales. Según las escasas fuentes documentales, en el año 804 se inició una repoblación de la zona, mediante el sistema de presuras, bajo la dirección del obispo de Valpuesta. En el año 957 la zona pasó a depender del monasterio de San Millán de la Cogolla y el río Ebro se convirtió en un eje vertebrador entre los territorios de Burgos, Álava y La Rioja como una frontera y base para las aspiraciones castellanas al reino de Navarra (Moratinos García, 2020). Es posible que algunos de los adultos fallecidos y enterrados allí fueran recién llegados ligados con migraciones o personas que no crearan familias, por ejemplo religiosos, y ello también podría justificar el número elevado de adultos en relación a los inmaduros. En este sentido es de esperar que los futuros análisis de isótopos de oxígeno puedan aportar información al respecto.

Otro factor, que se puede tener en cuenta en relación al número elevado de adultos respecto a niños y jóvenes, es un posible caso de mortalidad que hubiera afectado a un sector concreto de la población. Podría ser, por ejemplo, alguna acción militar que hubiera supuesto el fallecimiento de varones en el entorno y su enterramiento en este cementerio. Es cierto que figuran más varones que mujeres en el cementerio, pero no hay indicios de lesiones vinculadas claramente con acciones intencionales. Aunque tampoco sería descartable una mayoría de varones y una escasez de niños en un entorno fronterizo sin que se llegue a la antigua interpretación de Alberto del Castillo (1972) de considerar este cementerio como el de una población de "campesinos-soldados".

En cuanto a la mortalidad de los adultos los resultados obtenidos son los propios de una población rural medieval, con una baja esperanza de vida y muy pocos sujetos que alcancen edades avanzadas. De hecho, la distribución es muy semejante a la de otras poblaciones medievales de la provincia de León (Candelas González et al., 2016; Caro y Sánchez-García, 2016; López, 2002). Es normal que la mayoría de mujeres falleciera durante su periodo fértil, pero no debe confundirse la mortalidad en este periodo con la mortalidad materna (Mafart, 1994) puesto que son muchas las causas que pueden afectar a la salud de las mujeres. En sociedades agrícolas prehistóricas la maternidad recaía en las mujeres muy jóvenes lo que causaba una elevada mortalidad entre estas (Chamberlain, 2006). En algunos cementerios medievales rurales se aprecia este fenómeno en el sentido de que ninguna o casi ninguna mujer superó los 30 años (Šlaus, 2000; Guede et al., 2017; Jiménez-Brobeil et al. 2020) pero en Tejuela son muy pocas las fallecidas antes de cumplir esta edad. Si tenemos en cuenta que figura una aparente baja fertilidad, es posible que las mujeres accedieran al matrimonio a una edad más elevada, hecho constatado en muchas poblaciones agrícolas preindustriales de las que se cuenta con datos demográficos y que constituía un método de control de natalidad para limitar el tamaño de la población según la disponibilidad de recursos del entorno (Flinn, 1989).

Si se compara la población de Tejuela con las también medievales de Palacios (Palacios de la Sierra, Burgos) y San Baudelio de Berlanga (Berlanga de Duero, Soria), estudiadas por los mismos autores y con idéntica metodología, se pueden constatar algunas de las hipótesis planteadas. En Palacios, una población más grande e importante (figura 7), donde presuntamente las condiciones de vida fueron mejores que en Tejuela (Castillo González, 2008) se observa una mayor supervivencia de varones con más de 41 años. Entre las mujeres (figura 8), en Palacios figura una distribución muy parecida a la de Tejuela con una elevada mortalidad de las menores de 40 años, pero, sin embargo, con la presencia de algunas que superaron los 61. Por otra parte, la granja de San Baudelio, en la que solo habitaron unas pocas familias (Jiménez-Brobeil et al., 2020), presenta una estructura demográfica diferente con una elevada mortalidad en ambos sexos y de forma dramática en las mujeres, donde ninguna superó los 30 años.

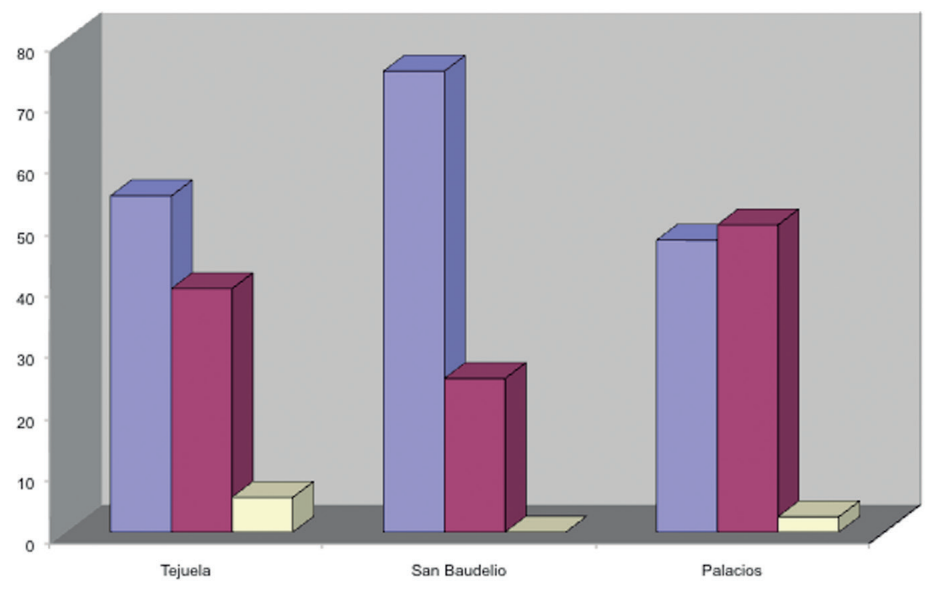

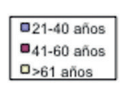

Fig.7. Comparación de la distribución de los individuos masculinos de Tejuela según clases de edad con la de los cementerios de San Baudelio de Berlanga y Palacios de la Sierra / Comparison of male individual distribution in Tejuela according to age classes with that of the cemeteries of San Baudelio de Berlanga and Palacios de la Sierra. 


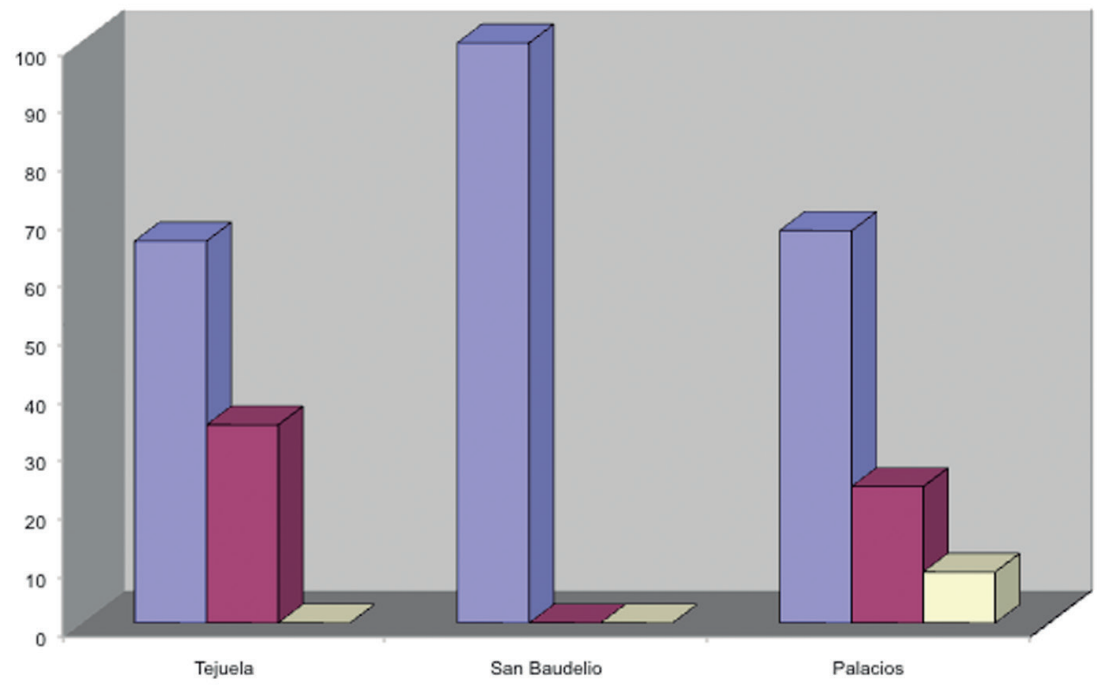

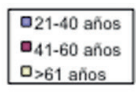

Fig.8. Comparación de la distribución de los individuos femeninos de Tejuela según clases de edad con la de los cementerios de San Baudelio de Berlanga y Palacios de la Sierra. / Comparison of female individual distribution in Tejuela according to age classes with that of the cemeteries of San Baudelio de Berlanga and Palacios de la Sierra.

\section{CONCLUSIONES}

El cementerio de Tejuela tuvo un periodo de uso corto, que no llegó a dos siglos, y muestra una distribución por grupos de edad que no coincide con la distribución de defunciones en una población histórica del régimen demográfico antiguo, principalmente porque faltan niños (Paine y Harpending, 1996). La escasez de niños de corta edad es un fenómeno común a muchas poblaciones arqueológicas, aunque en este caso el número de tumbas conservadas señalaría que hay otros factores aparte de los tafonómicos o metodológicos. El bajo índice de juventud (IJ), unido a la escasez de niños, apunta a una población con baja fertilidad. Este cementerio fue resultado de un movimiento fundacional en una zona fronteriza que tal vez atrajo a varios individuos adultos de los que algunos no llegaron a formar familias y eso, combinado con una posible menor mortalidad de los niños pequeños, podría justificar la distribución poblacional hallada. En cualquier caso, se trata de una población a la que, por su tamaño y distribución, no se pueden aplicar las clásicas fórmulas utilizadas en los estudios paleodemográficos. También resulta diferente a otras poblaciones medievales de la zona con índices de juventud muy altos. Las edades de defunción de los individuos adultos indicarían unas duras condiciones de vida, sobre todo entre las mujeres. De todos modos, hay que tener presente que la Paleodemografía es una disciplina que parte de premisas que muy difícilmente se cumplen y que realmente no puede solventar los problemas planteados por esta población sobre la que surgen muchas hipótesis, pero muy pocas conclusiones certeras.

\section{AGRADECIMIENTOS}

Este trabajo se ha realizado dentro del proyecto HAR2016-75788-P "Salud y alimentación en poblaciones rurales de la España medieval" financiado por el Gobierno de España.

\section{BIBLIOGRAFÍA}

Acsádi, G., Nemeskéri, J., 1970. History of Human Life Span and Mortality. Akademiai Kiado, Budapest.

Álvaro Rueda, K., Travé Allepuz, E., López Pérez, M.D., 2018. Excavaciones arqueológicas en el yacimiento altomedieval de Revenga: nuevos datos para el conocimiento de los espacios de hábitat altomedieval en el Alto Arlanza (Burgos). Territorio, Sociedad y Poder 13, 5-21.

Aratikos Arqueólogos, 2010. Excavación arqueológica en la necrópolis de Santa María de Tejuela en Villanueva Soportilla (Bozóo, Burgos). Diputación de Burgos, Burgos.

Barbiera, I., Dalla-Zuanna, G., 2009. Population dynamics in Italy in the Middle Ages: new insights from archaeological findings. Population and development Review 35, 367-389.

Bello, S.M., Thomann, A., Signoli, M., Dutour, O., Andrews, P., 2006. Age and sex bias in the reconstruction of past population structures. American Journal of Physical Anthropology 129, 24-38.

Bocquet-Appel, J.P., 2002. The paleoanthropological traces of Neolithic demographic transition. Current Anthropology 43, 638-650.

Bocquet-Appel, J.P., Bacro, J.N., 2008. Estimation of an age distribution with its confidence intervals using an iterative Bayesian procedure and a bootstrap sampling approach. In: Bocquet-Appel, J.P. (Ed.), Recent advances in Palaeodemography. Data, Techniques, Patterns, 63-82. Springer, Dordrecht.

Bocquet-Appel, J.P., Naji, S., 2006. Testing the hypothesis of a worldwide Neolithic demographic transition: corroboration from American cemeteries. Current Anthropology 47, 341-365.

Buchet, L., 1983. L'inhumation en basiliques funéraires. Observations, interprétations et commentaires. En: Buchet, L. (Ed.), Le matériel anthropologique provenant des édifices religieux, 69-73. C.N.R.S., Paris.

Buikstra, J., Ubelaker, D., 1994. Standards for data collection from human skeletal remains. Arkansas Archeological Survey Research, Fayetteville.

Byers, S., 2005. Introduction to Forensic Anthropology. Allyn and Bacon, Boston. 
Candelas González, N., Núñez Cantalapiedra, A., Rascón Pérez, J., Cambra-Moo, O., Muñoz Villarejo, F., Campomanes Alvaredo, E., Gutiérrez González, J.A., González Martín, A., 2016. Características paleodemográficas de la población recuperada del cementerio de Marialba de la Ribera (Villaturiel, León, España) (s.IV-XIII). Munibe. Antropologia-Arkeologia 67, 151-166.

Caro Dobón, L., Sánchez-García, E., 2016. Antropología física de poblaciones históricas de Castilla y León. En: Quirós Castillo, J.A. (dir.), Demografía, Paleopatologías y desigualdad social en el noroeste peninsular en época medieval, 97-121. Universidad del País Vasco, Bilbao.

Castillo, A. del., 1972. Excavaciones altomedievales en las provincias de Soria, Logroño y Burgos. Excavaciones Arqueológicas en España, Madrid.

Castillo González, C., 2008. Evolución de los estados de salud-enfermedad de poblaciones medievales del Alto Ebro y Alto Duero. Tesis Doctoral, Granada: Universidad de Granada, Granada. Disponible en: https://hera.ugr.es/tesisugr/17381824.pdf

Chamberlain, A., 2006. Demography in Archaeology. Cambridge University Press, Cambridge.

Cipriano de Cartago, 1998. Cartas. Carta 64, 306-311. Biblioteca Clásica Gredos, Madrid.

Flinn, M.W., 1989. El Sistema demográfico europeo. 15001820. Crítica, Barcelona.

Gélis, J., 2006. Les enfants des limbes. Mort-nés et parents dans l'Europe chrétienne. Audibert, Paris.

González Martín, A., Rascón Pérez, J., Cambra-Moo, O., Pimentel de Francisco, G., Campo Martín, M., 2016. Estudio poblacional del cementerio medieval de Veranes (Gijón, Asturias): estado de la cuestión. En: Quirós Castillo, J.A. (dir.), Demografía, Paleopatologías y desigualdad social en el noroeste peninsular en época medieval, 83-95. Universidad del País Vasco, Bilbao.

Guede, I., Ortega, L.A., Zuluaga, M.C., Alonso-Olazabal, A., Murelaga, X., Solaun, J.L., Sánchez, I., Azkarate, A., 2017. Isotopic evidence for the reconstruction of diet and mobility during village formation in the Early Middle Ages: Las Gobas (Burgos, northern Spain). Archaeological and Anthropological Sciences. https:/doi.org/10.1007/s12520-017-0510-9.

Hoppa, R.D., Vaupel, J.W., 2002. Paleodemography: Age Distributions from Skeletal Samples. Cambridge University Press, Cambridge.

Jackes, M.K., 2000. Building the bases for paleodemographic analysis: adult age determination. En: Katzemberg, M.A., Saunders, S.R., (Eds.), Biological Anthropology of the Human Skeleton, 417-466. Wiley-Liss, New York.

Jackes, M.K., 2011. Representativeness and Bias in Archaeological Skeletal Samples. In: Agarwal, S.C., Glencross, B.A. (Eds.), Social Bioarchaeology, 109-146. Wiley-Blackwell, Malden.

Jiménez-Brobeil, S.A., Maroto, R.M., Laffranchi, Z., Roca, M.G., Granados Torres, A., Delgado Huertas, A., 2020. Exploring diet in an isolated medieval rural community of Northern Iberia: the case study of San Baudelio de Berlanga (Soria, Spain). Journal of Archaeological Science: Reports. https://doi.org/10.1016/j. jasrep.2020.102218

Lewis, M.E., 2007. The Bioarchaeology of Children. Cambridge University Press, Cambridge.
Livi-Bacci, M., 1999. Historia mínima de la población mundial. Ariel, Barcelona.

López Martínez, B., 2002. Los pobladores del antiguo reino de León. Universidad de León, León.

López Pérez, M.D., Álvaro Rueda, K., Travé Allepuz, E., 2016. Rock-cut cemeteries and settlement processes at the Upper Arlanza basin (Burgos, Pain): a Late Antique and Early Medieval landscape analysis. Zephyrus LXXVIII, 173-191.

Mafart, Y., 1994. Approche de la mortalité maternelle au Moyen Âge en Provence. En: Buchet, L. (Ed.), La femme pendant le Moyen Âge et l'Époque Moderne, 207-219. CNRS, Paris.

Maroto Benavides, R.M., 2004. Antropología de las poblaciones femeninas medievales del Alto Ebro y Alto Duero. Universidad de Granada, Granada.

Martín Alonso, J.F., Maroto, R.M., Jiménez-Brobeil, S.A. (en prensa). Esqueletos inhumados en cementerios excavados en roca: el caso de Tejuela/Villanueva de Soportilla (Burgos). Archivo Español de Arqueología.

Martín Viso, I., 2012. Enterramientos, memoria social y paisaje en la Alta Edad Media: propuestas para un análisis de las tumbas excavadas en roca en el centro-oeste de la Península Ibérica. Zephyrus LXIX, 165-187.

Milner, G.R., Wood, J.W., Boldsen, J.L., 2008. Advances in Paleodemography. In: Katzemberg, M.A., Saunders, S.R. (Eds.), Biological Anthropology of the Human Skeleton, 561-600. John Willey and Sons, New Jersey.

Milner, G.R., Boldsen, J.L., 2012. Estimating age and sex from the skeleton, a paleopathological perspective. En: Grauer, A.L. (Ed.), A Companion to Paleopathology, 268-284. Wiley-Blackwell, Chichester.

Minvielle, S., 2007. Les crises de mortalité dans le Sud-ouest Aquitain de la fin du XVIle au milieu du XIXe siècle. In: Castex, D., Carton, I. (Eds.), Épidémies et crises de mortalité du passé, 51-76. Ausonius, Bordeaux.

Moratinos García, M., 2020. Contexto histórico. Referencias documentales escritas- En: Palomino, A.L., Negredo, M. (Coords.) La comunidad aldeana de Tejuela en época medieval. Arqueología funeraria y doméstica en el Alto Valle del Ebro (en prensa). Diputación de Burgos, Burgos.

Müller, H.G., Love, B., Hoppa, R.D., 2002. A semiparametric method for estimating demographyc profiles from age indicator data, American Journal of Physical Anthopology 117, 1-14.

Padilla, J.I., Álvaro-Rueda, K., 2010. Necrópolis rupestres y el poblamiento altomedieval en el alto Arlanza (Burgos). En la España Medieval 33, 259-294

Padilla, J.I., Álvaro-Rueda, K., 2013. Asentamientos altomedievales del Alto Arlanza (Burgos). El despoblado medieval de Revenga. Pyrenae 44, 11-41.

Paine, R.R., Harpending, H.C., 1996. Assessing the reliability of paleodemographic fertility estimators using simulated skeletal distributions. American Journal of Physical Anthropology 101, 151-159.

Palomino Lázaro, A.L., Negredo García, M. (Coords). 2020. La comunidad aldeana de Tejuela en época medieval. Arqueología funeraria y doméstica en el Alto Valle del Ebro, en prensa. Diputación de Burgos, Burgos.

Pfeiffer, S., Doyle, L.E., Kurki, H.K., Harrington, L., Ginter, J.K., Merritt, C.E., 2014. Discernment of mortality risk associated with childbirth in archaeologically derived forager skeletons. International Journal of Paleopathology 7, 15-24. 
Quirós, J.A., 2006. La génesis del paisaje medieval en Álava: la formación de la red aldeana. Arqueología y Territorio Medieval 13, 49-94.

Quirós, J.A., 2011. L'eccezione che confrema la regola? Incastellamento nella valle dell'Ebro nel X secolo: il castello di Treviño. Archeologia Medievale XXXVIII, 113-136.

Quirós, J.A., 2013. Los comportamientos alimentarios del campesinado medieval en el País Vasco y su entorno (siglos VIII-XIV). Historia Agraria 59, 13-41.

Sánchez-Albornoz, C., 1966. Despoblación y repoblación del Valle del Duero. Instituto de Historia de España, Buenos Aires.

Saunders, S.R., Hoppa, R.D., 1993. Growth deficit in survivors and non-survivors: Biological mortality bias in sub-adult skeletal samples. Yearbook of Physical Anthropology 36, 127-151.

Scheuer, L., Black, S., 2000. Developmental Juvenile Osteology. Academic Press, San Diego.

Séguy, I., Buchet, L., Belaigues-Rossard, M., Couvert, N., Perraut, C., 2006. Des tables types de mortalité pour les populations pré-industrielles. Présentation, discussion et applications. In: Buchet, L., Dauphin, C., Séguy, I. (Eds.), La paléodémographie. Mémoire d'os, mémoire d'hommes, 303-321. APDCA, Antibes.

Séguy, I., Buchet, L., Bringé, A., Belaigues-Rossard, M., Beurnier, P., Couvert, N., Perraut, C., 2008. Model Life Tables for Pre-Industrial Populations: First Application in Palaeodemography. In: Bocquet-Appel, J.P. (Ed.), Recent advances in Palaeodemography. Data, Techniques, Patterns, 83-117. Springer, Dordrecht.

Šlaus, M., 2000. Biocultural analysis of sex differences in mortality profiles and stress levels in the late medieval population from Nova Raca, Croatia. American Journal of Physical Anthropology 111, 193-209.
Souich, Ph. du, Botella, M., Ruíz, L., 1991. Antropología de la población medieval de Villanueva de Soportilla (Burgos). Antropología y Paleoecología Humana 6, 57-83.

Suby, J., Luna, L., Aranda, C., Flensborg, G., 2017. First approximation to paleodemography through age-at-death profiles in hunter-gatherers from Southern Patagonia during middle-late Holocene. Quaternary International 438, 174-188.

Tente, C., 2015. Tumbas rupestres en el Alto Mondego (Guarda, Portugal). Patrones de distribución, significados y construcción del paisaje rural altomedieval. Munibe. Antropologia-Arkeologia 66, 271-290.

Usher, B.M., 2002. Reference samples: the first step in linking biology and age in the human skeleton. In: Hoppa, R.D., Vaupel, J.W. (Eds), Paleodemography: Age Distributions from Skeletal Samples, 29-47. Cambridge University Press, Cambridge.

Waldron, T., 1994. Counting the Dead: The Epidemiology of Skeletal Populations. Willey, Chichester.

Waldron, T., 2007. Palaeoepidemiology: The measure of disease in the human past. Left Coast Press Inc, Walnut Creek.

Wittwer-Backofen,U., Buckberry, J., Czametzki, A., Doppler, S., Grupe, G., Hotz, G., Kemkes, A., Spencer Larsen, C., Prince, D., Wahl, J., Fabig, A., Weise, S. 2008. Basics in Paleodemography: a comparison of age indicators applied to the Early Medieval skeletal sample of Lauchheim. American Journal of Physical Anthropology 137, 384-396.

Wright, L.E., Yoder, C.J., 2003. Recent progress in Bioarchaeology: Approaches to the osteological paradox, Journal of Archaeological Research 11, 43-70. 
